Journal de la Société des américanistes

Journal de la Société

des américanistes

tome $93, \mathrm{n}^{\circ} 1$

\title{
Les répertoires graphiques amazoniens
}

Pierre Déléage

\section{OpenEdition}

Journals

Édition électronique

URL : https://journals.openedition.org/jsa/6693

DOI : 10.4000/jsa.6693

ISSN : 1957-7842

Éditeur

Société des américanistes

Édition imprimée

Date de publication : 1 décembre 2007

Pagination : 97-126

ISSN : 0037-9174

\section{Référence électronique}

Pierre Déléage, «Les répertoires graphiques amazoniens », Journal de la Société des américanistes [En ligne], 93-1 | 2007, mis en ligne le 15 juin 2012, consulté le 02 septembre 2022. URL : http:// journals.openedition.org/jsa/6693; DOI : https://doi.org/10.4000/jsa.6693 


\title{
LES RÉPERTOIRES GRAPHIQUES AMAZONIENS
}

\author{
Pierre DÉLÉAGE *
}

Cet article propose, dans un premier temps, une description détaillée des peintures faciales des Sharanahua, peuple pano d'Amazonie occidentale. Ces peintures sont réalisées à partir d'un répertoire graphique comprenant une série de motifs associés chacun à un nom spécifique. Sont présentés les principes de la composition de ces motifs, le sémantisme de leurs noms (dont la valeur est mnémotechnique), la manière dont ils sont utilisés pour former des peintures faciales et pour rendre visibles des relations sociales. Ce répertoire graphique est ensuite comparé à ceux d'autres cultures du bassin amazonien afin de mettre en évidence une catégorie homogène distincte de celle réunissant les figurations. [Mots clés : peinture corporelle, iconographie, mémoire, écriture, Sharanahua, Pano, Amazonie.]

Amazonian graphic repertoires. This paper begins with a description of the facial paintings of the Sharanahua, a Panoan people of Western Amazonia. The paintings are created based on a graphic repertoire comprising a series of motifs associated with a specific name. This study presents the principles of composition of these patterns, the mnemotechnical meaning of their names, and the ways they are utilized to create new paintings and make social relationships visible. This repertoire is then compared to those of other Amazonian cultures in order to show evidence for the existence of a homogenous category distinct from that joining together the figurations. [Key words : body painting, iconography, memory, writing, Sharanahua, Pano, Amazon.]

Repertorios gráficos amazónicos. Este artículo propone, inicialmente, una descripción detallada de las pinturas faciales de los sharanahua, un pueblo pano de la Amazonía occidental. Las pinturas son realizadas a partir de un repertorio gráfico que comprende una serie de motivos asociados a un nombre específico. Son presentados los principios de la composición de estos motivos, el significado mnemotécnico de sus nombres, la manera en que son utilizados para formar nuevas pinturas faciales y para rendir visibles las relaciones sociales. El repertorio gráfico es entonces comparado a aquellos de otras culturas de la cuenca amazónica con el fin de poner de relieve una categoría homogénea distinta de la que reúne las figuraciones. [Palabras claves : pintura corporal, iconografía, memoria, escritura, sharanahua, pano, Amazonía.]

* Laboratoire d'anthropologie sociale, 52 rue du Cardinal Lemoine, 75005 Paris/Department of Anthropology, UC Berkeley, 232 Kroeber Hall, Berkeley CA 94720, États-Unis [deleagepierre@hotmail.com].

Journal de la Société des Américanistes, 2007, 93-1, pp. 97-126. (O Société des Américanistes. 
La divination Ifa consiste en une série de techniques qui permettent d'engendrer, d'une manière apparemment aléatoire, une configuration parmi les 256 possibles. [...]

Le babalasto obtient ce résultat en lançant seize noix huit fois à la suite [...]. Les configurations qui en résultent sont nommées en yoruba des oddu, et en espagnol des signos ou des letras (Holbraad 2003, p. 52. Traduction de l'auteur).

Les procédures mises en cuvre par les devins pour construire les présages sont en tous points similaires à celles auxquelles les inventeurs de l'écriture eurent recours, au $\mathrm{IV}^{\mathrm{e}}$ millénaire, pour créer leur grammaire de signes ! Ceux-ci commencèrent, en effet, par imaginer une gamme de signes premiers qu'ils manipulèrent pour fabriquer des signes dérivés. Ils jouèrent alors de leurs dimensions, de leur disposition relative sur le support, selon qu'ils étaient dessinés au miroir, inclinés à droite ou à gauche, ou encore inachevés. Ils les agrémentèrent de surcharges, des hachures, des enchevêtrements de traits ou des pointillés, ou les associèrent par doublement ou triplement, parfois en position croisée, ou imbriqués les uns dans les autres. Ils associèrent également deux ou plusieurs signes premiers différents qui pouvaient, en outre, être augmentés de surcharges (Glassner s. d.).

Qui feuillette un des nombreux catalogues d'exposition consacrés à l'art amazonien, découvre, pêle-mêle, des ustensiles quotidiens décorés, des œuvres de plumasserie, des masques, des objets rituels liés au chamanisme, des dessins que l'on qualifie de «spontanés » ou encore des photographies de corps ornés de peintures au génipa. Le lecteur a alors l'impression d'un rassemblement arbitraire d'artefacts que seul un concept exogène vient légitimer - celui d'« art » dont la pertinence, une fois que l'on sait qu'il n'a pas vraiment d'équivalent dans les cultures amazoniennes, ce que tous les ouvrages de ce genre précisent d'une manière ou d'une autre, finit par paraître douteuse.

Si l'on veut vraiment définir les catégories adéquates permettant de classer; puis d'étudier dans toute leur complexité ces objets hétéroclites, il convient de mettre en place deux axes de recherche. Le premier consiste à se demander comment les peuples qui fabriquent et utilisent ces artefacts les catégorisent. Le second prend pour point de départ la description détaillée d'une de ces catégories, chez un peuple donné, et pour horizon sa comparaison avec son équivalent au sein d'autres peuples du bassin amazonien. Cet article souhaite mettre à l'épreuve ces méthodes de recherche.

Pour ce faire, nous nous appuierons sur la culture graphique des Sharanahua, peuple pano d'Amazonie occidentale, dont nous avons acquis une connaissance de première main au cours de ces dernières années. Les Sharanahua classent leurs images en deux catégories exclusives, au moins : les figurations et les dessins.

Pour les figurations, les Sharanahua disposent du terme yoshi qui permet de regrouper une série de phénomènes perceptifs qui sont, certes, hétérogènes, mais qui partagent une propriété : ils entretiennent une relation de figuration avec une autre entité. Le yoshi apparaît comme le double ou la représentation d'un être quelconque. Ainsi, une « ombre » ou un " reflet » sont des yoshi dans la mesure où ils ne sont pas perçus comme des entités naturelles, mais comme des phéno- 
mènes optiques que l'on pourrait qualifier de figuratifs ; ils ne font que dériver d'autres êtres qui, quant à eux, sont les modèles de ces figurations. Par ailleurs, une des acceptions du terme furoyoshi, où furo signifie « œil », est le reflet de soi dans la pupille de quelqu'un d'autre. De plus, les masques sont nommés fuso yoshi, où fuso signifie " visage "; ce faisant, les Sharanahua, qui ne possèdent pas (ou plus) de masques rituels, ne se réfèrent qu'au fait que les masques sont des figurations des visages humains. Enfin, le terme yoshi a été récemment utilisé par les Sharanahua pour désigner les « photographies ». Le champ sémantique de la catégorie de yoshi, malgré son hétérogénéité, réunit donc l'ensemble des phénomènes figuratifs connus dans cette culture. Parmi ceux-ci, deux phénomènes peuvent être considérés comme des productions iconographiques : le masque et la photographie. Une étude détaillée de la logique sémantique sous-jacente à la notion de yoshi dépasserait de loin les limites de ce travail ${ }^{1}$; ce qui importe pour notre propos, c'est qu'il s'agit là d'une première catégorie d'artefacts culturels.

Un autre terme sharanahua permet de désigner un genre d'image qui contraste totalement avec la catégorie des figurations. Il s'agit du mot cudu qui peut être traduit, bien imparfaitement, par « dessin ». Il se réfère prioritairement aux peintures faciales composées de divers motifs graphiques. Mais, de même que le terme yoshi en est venu à désigner les " photographies ", cudu signifie désormais " écriture ». Il s'agit donc là d'une seconde catégorie d'artefacts culturels ; nous proposons de la nommer, pour des raisons qui seront présentées au cours de cet article, " répertoire graphique ".

Les Sharanahua classent ainsi certaines de leurs images au sein de deux catégories exclusives: les figurations (yoshi) et les répertoires graphiques (cudu $)^{2}$. Le premier objectif de cet article est de fournir une description détaillée du répertoire graphique des Sharanahua, leur seconde catégorie d'artefacts. À partir de cette description, nous montrerons que cette catégorie est valide pour la plupart des cultures du bassin amazonien et ce, jusque dans ses traits les plus précis.

\section{Les Sharanahua}

Les Sharanahua parlent une langue pano et comptent près de 650 membres. Ils vivent au sein de villages d'environ 150 habitants chacun, dispersés sur les rives péruviennes du fleuve Purus, au cœur du bassin amazonien. Ils ont pour voisins d'autres groupes pano, tels que les Mastanahua, les Amahuaca ou les Cashinahua, mais aussi les Madihá (Culina), de langue arawa, ou les Yine (Piro), de langue arawak. Les informations présentées ici proviennent d'un seul village du Purus, dénommé Gasta Bala, où nous avons séjourné pendant 19 mois, échelonnés d'octobre 2001 à août 2004. Les Sharanahua, originaires des aftluents du Jurua, sont arrivés dans la région vers le milieu du siècle dernier. Ils vivent 
aujourd'hui, pour l'essentiel, de l'horticulture du manioc et de la banane plantain, associée à des activités de chasse et de pêche, hautement valorisées.

Il n'existe, à ce jour, que peu de documentation sur leurs peintures faciales. Dans la littérature ethnographique, les Sharanahua sont souvent considérés comme un peuple sans iconographie, sans dessins traditionnels. Ce point de vue est probablement celui des Shipibo-Conibo ou des Yine de l'Urubamba, dont les traditions graphiques sont mieux connues. Leurs ethnologues l'ont adopté sans tenir compte du travail pionnier d'Adolfo Torralba (1966; 1986), missionnaire dominicain qui, dès les années 1960, avait proposé une première description des peintures faciales sharanahua. Nous aurons l'occasion de revenir sur ses travaux. Une comparaison rapide entre le répertoire graphique qu'il a observé et celui qui est présenté ici permet de soutenir que cette tradition s'est très fidèlement transmise au cours des quarante dernières années.

\section{LA CONSTRUCTION DES MOTIFS}

L'étude des peintures corporelles ne peut se limiter à la simple observation d'œuvres achevées. De même que l'analyse des brouillons d'un écrivain est susceptible de rendre plus rigoureuse l'appréhension de ses textes, la description des peintures faciales des Sharanahua doit passer par l'observation de la façon dont elles sont effectuées.

Les peintures sharanahua sont réalisées pour la plupart au génipa (Genipa americana ; sharanahua : dadu), même si, quelquefois, du roucou (Bixa orellana ; sharanahua : chashca) ou du maquillage (violet ou rouge) acquis dans la petite ville métisse de Puerto Esperanza peuvent faire l'affaire. La pointe d'un morceau séché d'une feuille de palmier, empruntée la plupart du temps au toit de la maison, est enduite de l'une de ces substances afin de servir de pinceau. Souvent, une personne en peint une autre ; parfois on utilise un miroir pour dessiner sur soi ses propres peintures.

Un jour que j'observai un petit groupe de femmes se peignant les unes les autres, j'eus la surprise d'observer leur manière, tout à fait inattendue, de dessiner des croix et des carrés. Je pensai d'abord à une incongruité ; toutefois, ma curiosité ayant été attisée, je multipliai par la suite les observations jusqu'à me rendre compte qu'il s'agissait là d'une technique stable, partagée par l'ensemble des peintres sharanahua.

Ainsi, plutôt que de dessiner une croix par l'intersection, en leur milieu, de deux traits identiques, les Sharanahua tracent d'abord un chevron auquel ils accolent par la suite son symétrique en reflet (Figure 1) ${ }^{3}$. Le carré, quant à lui, n'est pas réalisé à l'aide d'un trait continu, mais résulte de l'opération inverse de symétrie appliquée à ces mêmes chevrons (Figure 2). Une fois isolé, ce simple motif, pour lequel les Sharanahua n'ont pas de mot particulier, permet de 


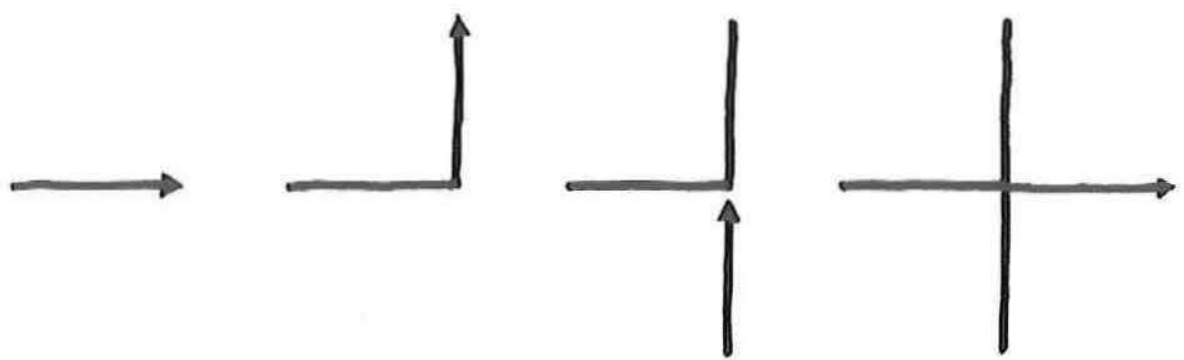

FIG. 1 - Comment les Sharanahua dessinent-ils une « croix "?
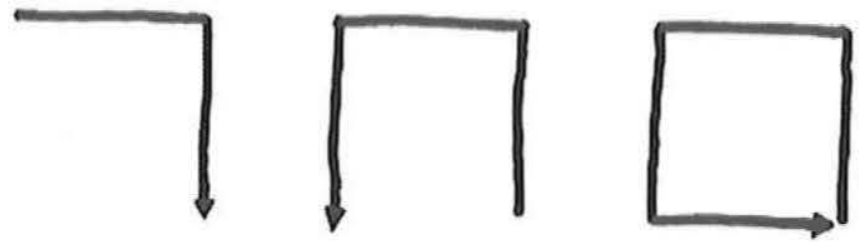

FIG. 2 - Comment les Sharanahua dessinent-ils un " carré »?

reconstruire tous les motifs de leur répertoire graphique (Figure 3). On obtient là quelque chose comme un motif originel, le chevron, à partir duquel sont formés plusieurs motifs de base qui, eux non plus, ne sont pas nommés : le zigzag, le triangle, le losange, le carré ou la croix. Ces éléments subiront ensuite une série de transformations et de variations jusqu'à engendrer la série de motifs nommés propre au répertoire graphique sharanahua. Ces opérations graphiques mettent en jeu les lois bien connues de la composition au sein desquelles on reconnaîtra la symétrie (en miroir ou inversée), la translation et la rotation (Washburn et Crowe 1988). L'important ici est de bien comprendre que la réalisation de ces motifs peut être pensée uniquement à partir de ces principes purement graphiques.

Ce phénomène d'engendrement des motifs du répertoire, à partir de motifs de base et suivant divers principes de symétrie, se retrouve chez de nombreux peuples amazoniens. Il est vrai que l'on trouve peu de motifs originels uniques ${ }^{4}$ au sein de ces diverses traditions graphiques ; néanmoins, la différence entre motifs de base et motifs composés, nommés, propres au répertoire graphique, peut s'y manifester de deux façons.

La première transparaît dans la manière dont certains peuples ont décidé de nommer ces motifs de base : il s'agit alors de noms techniques qui correspondent sémantiquement à des mots tels que "ligne », "croix », " angle », etc. Ainsi, selon Peter Roe (1980, p. 52), les Shipibo disposent de termes spécifiques pour se 


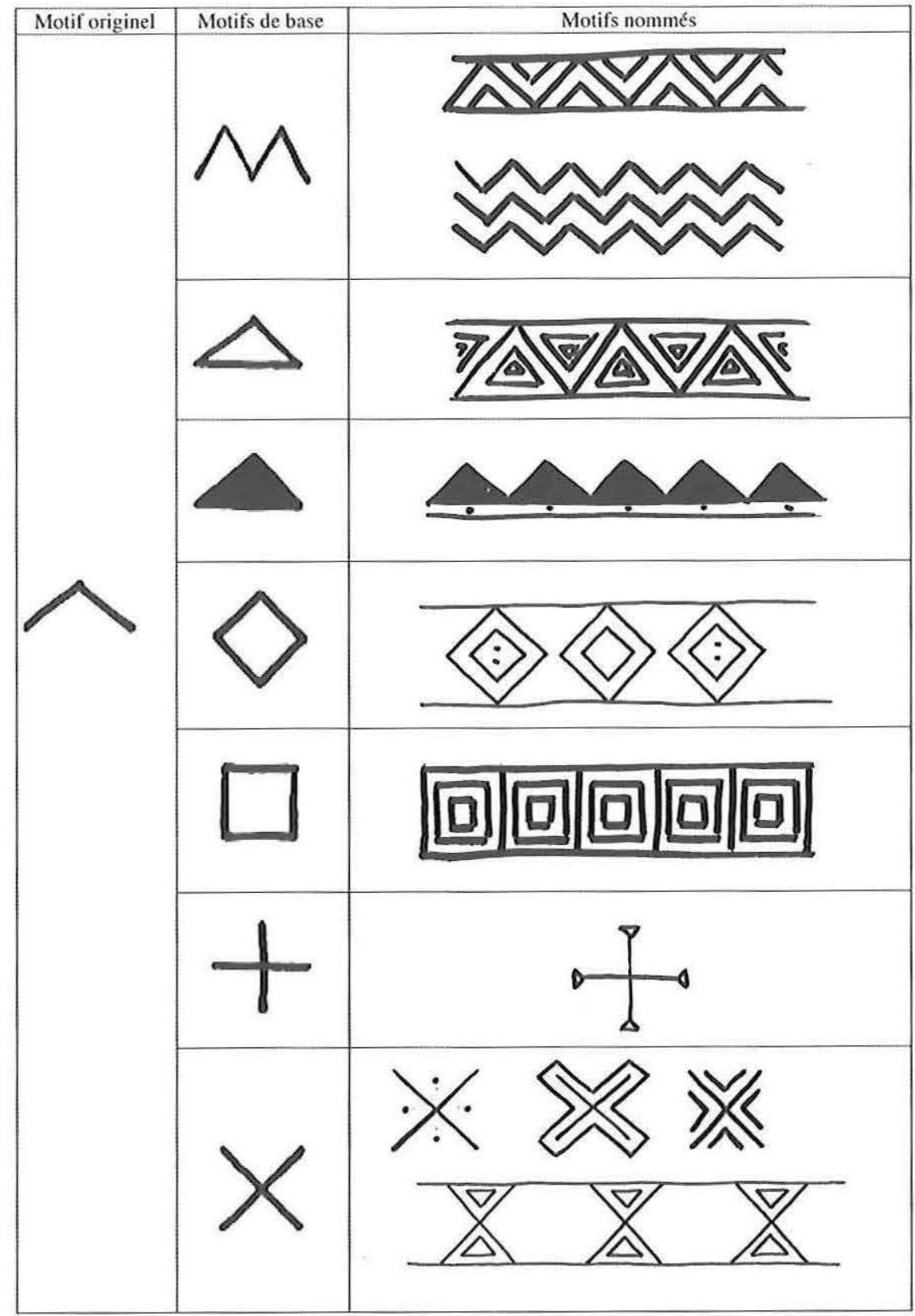

FIG. 3 - Le motif originel et l'engendrement des motifs sharanahua. 
référer aux motifs de base de leurs dessins (quënëa). Le « carré » est nommé toro, la « croix » ashta et le « zigzag » tsiton. Les motifs qui entrent dans la composition des dessins, mais auxquels ils ne se réduisent pas, sont donc ici, contrairement au cas sharanahua, nommés explicitement ${ }^{5}$. De même, selon Dominique Gallois (2002, p. 14), les Wajãpi utilisent quatre termes pour se référer aux composants des motifs du répertoire : wiriwiri signifie " pointillés », kã'gwer « lignes parallèles », rykyry « croix » et moj (ou moju) « zigzag ». Comme chez les Shipibo, ces motifs de base ne sont que des composants : leur assemblage permet d'engendrer l'intégralité du répertoire de motifs des Wajãpi ${ }^{6}$.

Certains ethnologues sont parvenus à reconstituer l'ensemble du répertoire graphique d'une culture en ayant recours aux seules lois de la composition : ce fait est à lui seul une seconde manifestation de la différence entre motifs de base et composés. On pourra constater ce résultat grâce à l'exemple visuel de la construction des motifs trumai (Figure 4), selon Aurore Monod Becquelin (1993, figures 84 et 85 ). Une analyse similaire a été proposée par Peter Roe (1980, p. 59) pour les peintures shipibo. Dans tous les cas, on peut affirmer que la construction d'un répertoire de motifs obéit à une logique graphique autonome fondée sur la présence d'une ou de plusieurs formes de base auxquelles sont appliquées les principales lois de la composition.

\section{LES MOTIFS DU RÉPERTOIRE GRAPHIQUE ET LEURS NOMS}

Quelle que soit la manière dont le répertoire est constitué, elle aboutit à une série de motifs nettement individualisés à laquelle correspond une série de noms. Les Sharanahua utilisent ainsi, pour réaliser leurs peintures faciales, une douzaine de motifs nommés (Figure 5). Que pouvons-nous dire d'une telle tradition graphique?

Il faut constater que l'on trouve ce type de répertoires chez la plupart des peuples du bassin amazonien. La stratégie d'interprétation la plus courante jusqu'à ce jour a été de considérer ces listes de motifs nommés comme des figurations. Ainsi, le motif "coude du singe-araignée » serait une figuration, métonymique certes, du singe-araignée réel. Et l'orientation de la recherche consisterait dès lors à relier chacun de ces motifs figuratifs à leur référent - quitte à explorer la mythologie afin de repérer divers traits pertinents conférant une valeur, plus ou moins schématique, à ces dessins. Cette stratégie n'a toutefois pas permis, nous semble-t-il, d'enrichir réellement notre compréhension des traditions graphiques amazoniennes. Nous pensons qu'il s'agit là d'une fausse piste.

Nous lui opposerons une autre approche, plus évidente, fondée sur la comparaison de plusieurs répertoires amazoniens de motifs nommés, du moins de ceux qui nous sont accessibles au sein de la littérature ethnologique. Pour entamer cette comparaison, nous souhaitons partager avec le lecteur un de nos étonnements les 

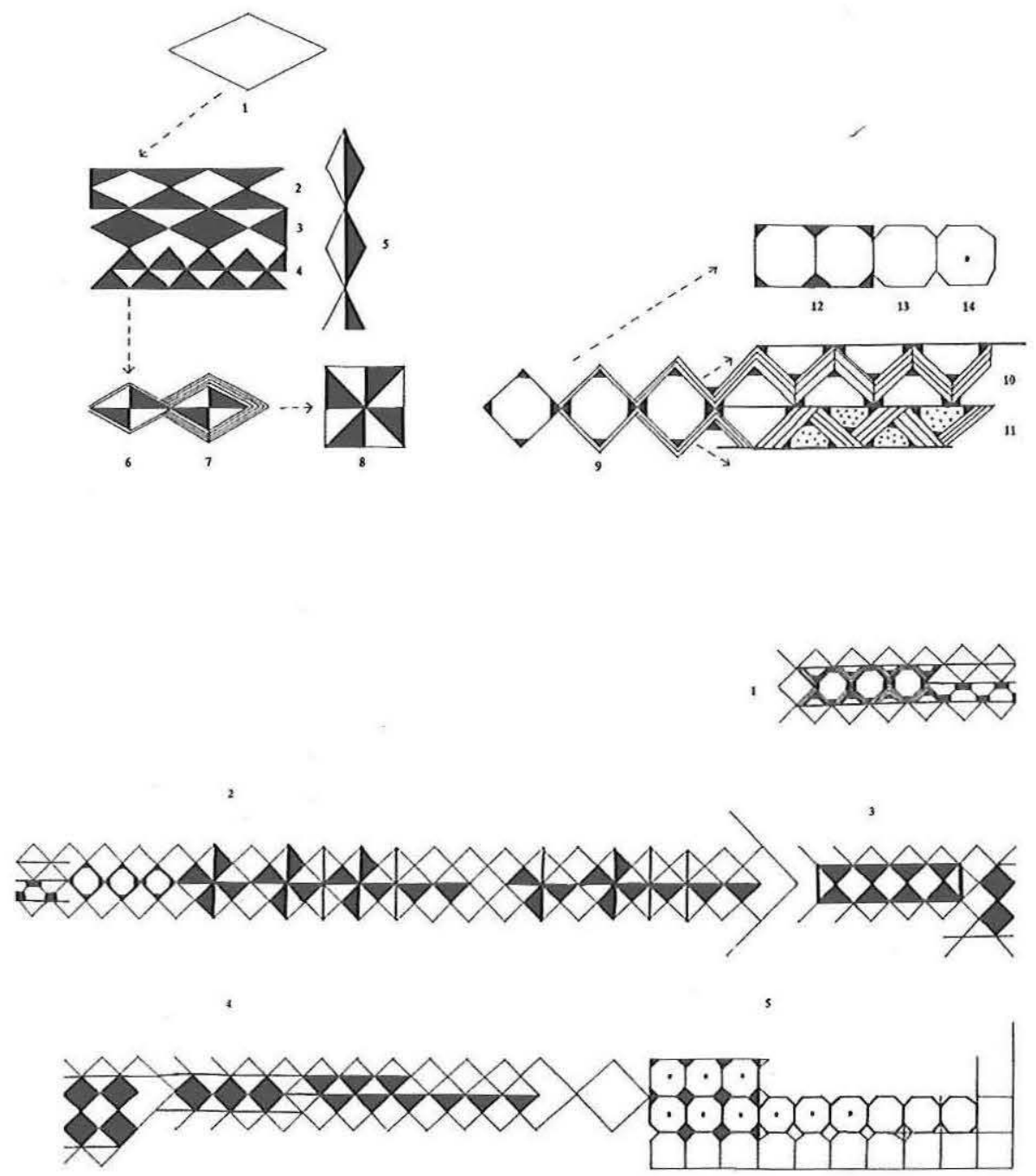

FIG. 4 - Engendrement logique des motifs trumai (Monod Becquelin 1993, figures 84 et 85 ).

plus durables. Pour cela, il faut nous référer à l'étude que Dominique Gallois $(1992 ; 2002)$ a réalisée à partir des motifs des Wajãpi, peuple de langue tupi, vivant de part et d'autre de la frontière délimitant la Guyane française du Brésil. Les Wajãpi disposent d'un répertoire d'une vingtaine de motifs nommés et certains sont utilisés beaucoup plus fréquemment que les autres (Gallois 1992, p. 212). Or si l'on juxtapose les répertoires sharanahua et wajãpi, en particulier les 


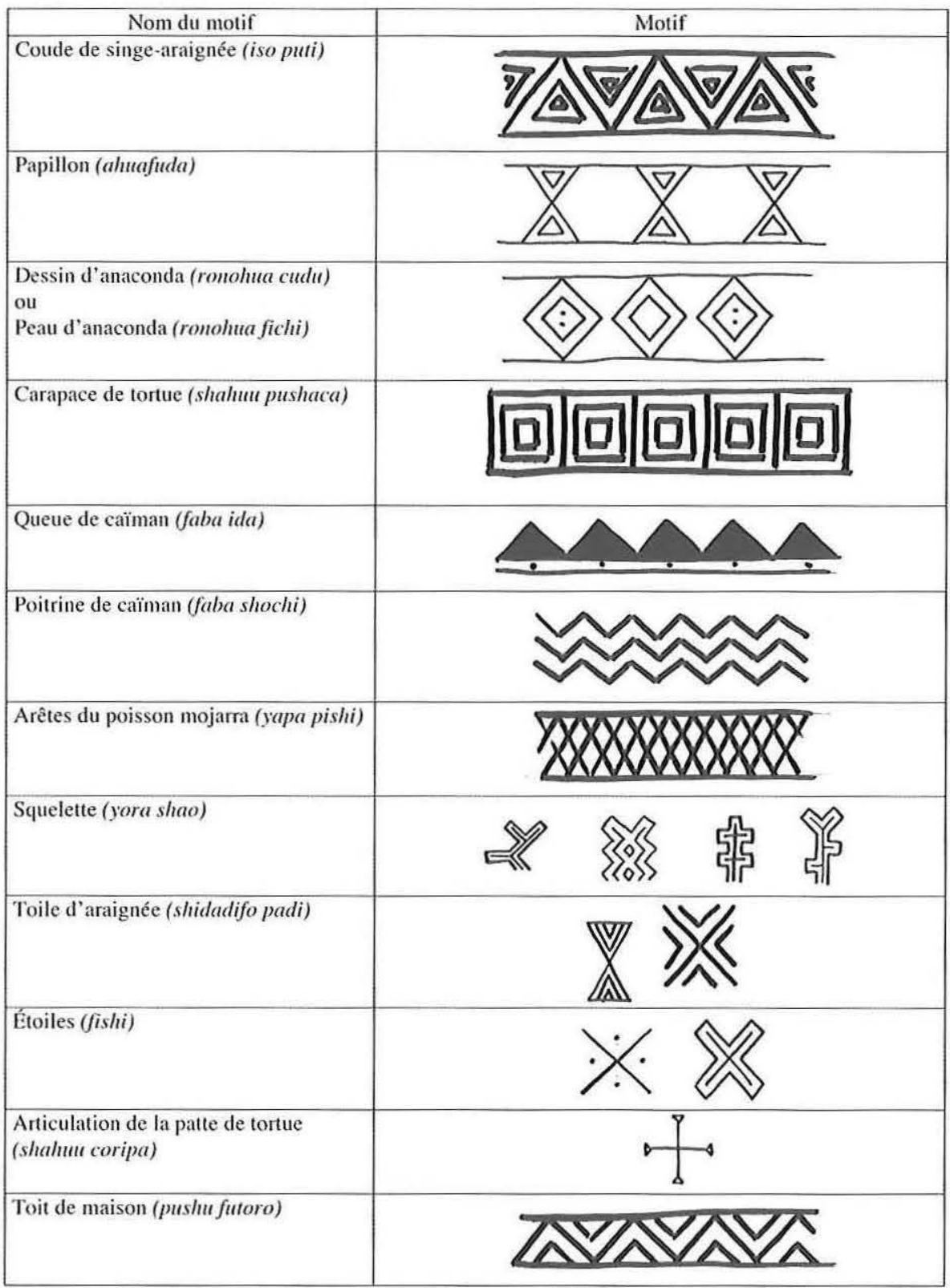

FIG. 5-Répertoire graphique des Sharanahua. 


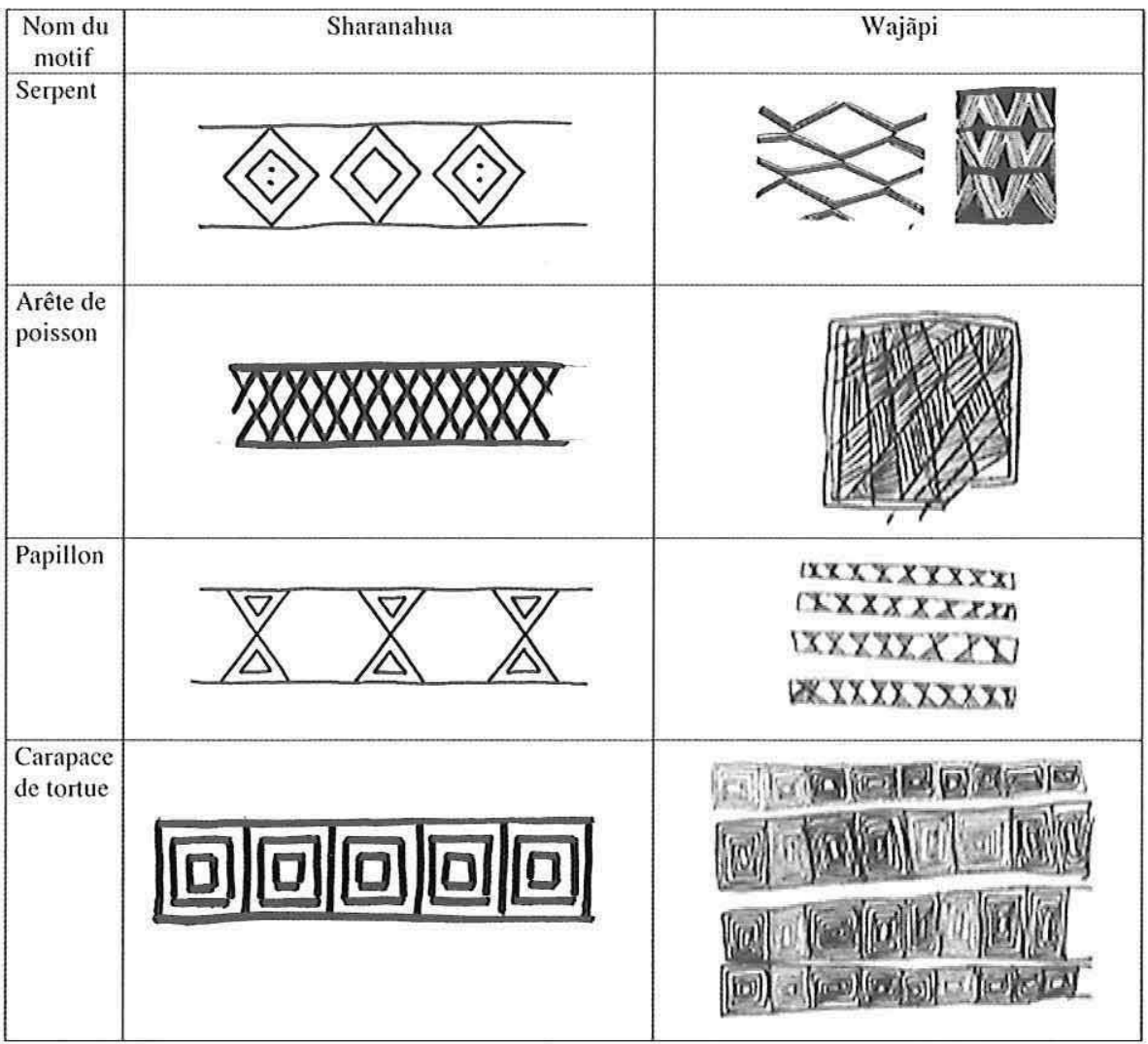

FIG. 6 - Comparaison entre les motifs nommés, sharanahua et wajāpi, les plus fréquents.

quatre motifs les plus fréquents, on pourra constater, avec surprise, une remarquable homogénéité (Figure 6). On observe, en effet, la même série de motifs associée à la même série de noms ${ }^{7}$.

Cette découverte nous a conduit à poursuivre la comparaison et à tenter d'évaluer, dans les limites du bassin amazonien, le degré d'homogénéité de cette relation entre certains motifs et certains noms. Pour ce faire, nous avons sélectionné un petit nombre de motifs nommés et sommes partis à la recherche de leur équivalent dans d'autres répertoires graphiques amazoniens ${ }^{8}$. Le résultat de cette investigation est éloquent : nous le soumettons au regard du lecteur dans le cadre de trois tableaux synthétiques. Le premier s'attache au motif " serpent» (Figure 7) qui oscille entre une série de zigzags (il s'agit alors plutôt de la forme du serpent) et une série de losanges (un dédoublement symétrique des séries de 


\begin{tabular}{|c|c|c|c|}
\hline Peuple & Références & Nom du motif & Motif \\
\hline Sharanahua & Données personnelles & $\begin{array}{l}\text { Dessin } \\
\text { d'anaconda }\end{array}$ & @ \\
\hline Sharanahua & Torralba 1966, p. 11 & $\begin{array}{l}\text { Queue } \\
\text { d'anaconda }\end{array}$ & \\
\hline Cashinahua & $\begin{array}{l}\text { Lagrou } 1998 \text { (A. Pinheiro Feitosa, } \\
\text { 20/08/1995) }\end{array}$ & Tête de serpent & \\
\hline Ashaninka & Rojas Zolezzi 2004 & Serpents & \\
\hline Matsiguenga & Casevitz 1980-1981, p. 288 (130R) & Serpent & \\
\hline Jivaro & $\begin{array}{l}\text { Karsten 1935, p. } 493 \text { (d, e) ; Pellizzaro } \\
\text { 1993, pp. 256-257 }\end{array}$ & Serpent & \\
\hline Yekuana & Guss 1989, p. 217 & $\begin{array}{l}\text { Traces de } \\
\text { serpent }\end{array}$ & \\
\hline Akawaio & Butt 1957, p. 43 & Serpent & \\
\hline Wajãpi & Gallois 1992 , p. $211 ; 2002$, pp. $18-19$ & Serpent & \\
\hline Karaja & Boas 2003, p. 119 (c) & $\begin{array}{l}\text { Tache de } \\
\text { serpent }\end{array}$ & \\
\hline Xerente & Lopes da Silva et Farias 1992, p. 99 & Anaconda & \\
\hline Kayapó & Vidal 1992, p. 151 (h), p. 163 (37) & $\begin{array}{l}\text { Vertèbre de } \\
\text { serpent }\end{array}$ & \\
\hline Bakairi & Von den Steinen 1940 & Serpent & \\
\hline Bakairi & Schmidt 1942 & Serpent & \\
\hline Wauja & Coelho Pentado 1993, pp. 614, 618 & Serpent & \\
\hline Wauja & Barcelos Neto 2002, p. 164 (11) & Boa & \\
\hline Yawalapiti & Ribeiro 1993, p. 587 & Serpent & \\
\hline Trumai & Monod Becquelin 1975; 1993, p. 514 & Boa, anaconda & \\
\hline Kuikoro & Franchetto 2003, p. 62 & Serpent & \\
\hline
\end{tabular}

Fig. 7 - Le motif « serpent ».

zigzags correspondant généralement aux dessins sur la peau du serpent) ${ }^{9}$. Le deuxième compare les motifs " tortue » ou " carapace de tortue ", dominés par les carrés souvent emboîtés les uns dans les autres (Figure 8) ${ }^{10}$. Le troisième regroupe les motifs « poisson » ou " arêtes de poisson », composés de multiples hachures parallèles disposées soit de part et d'autre d'un axe de symétrie, soit en 


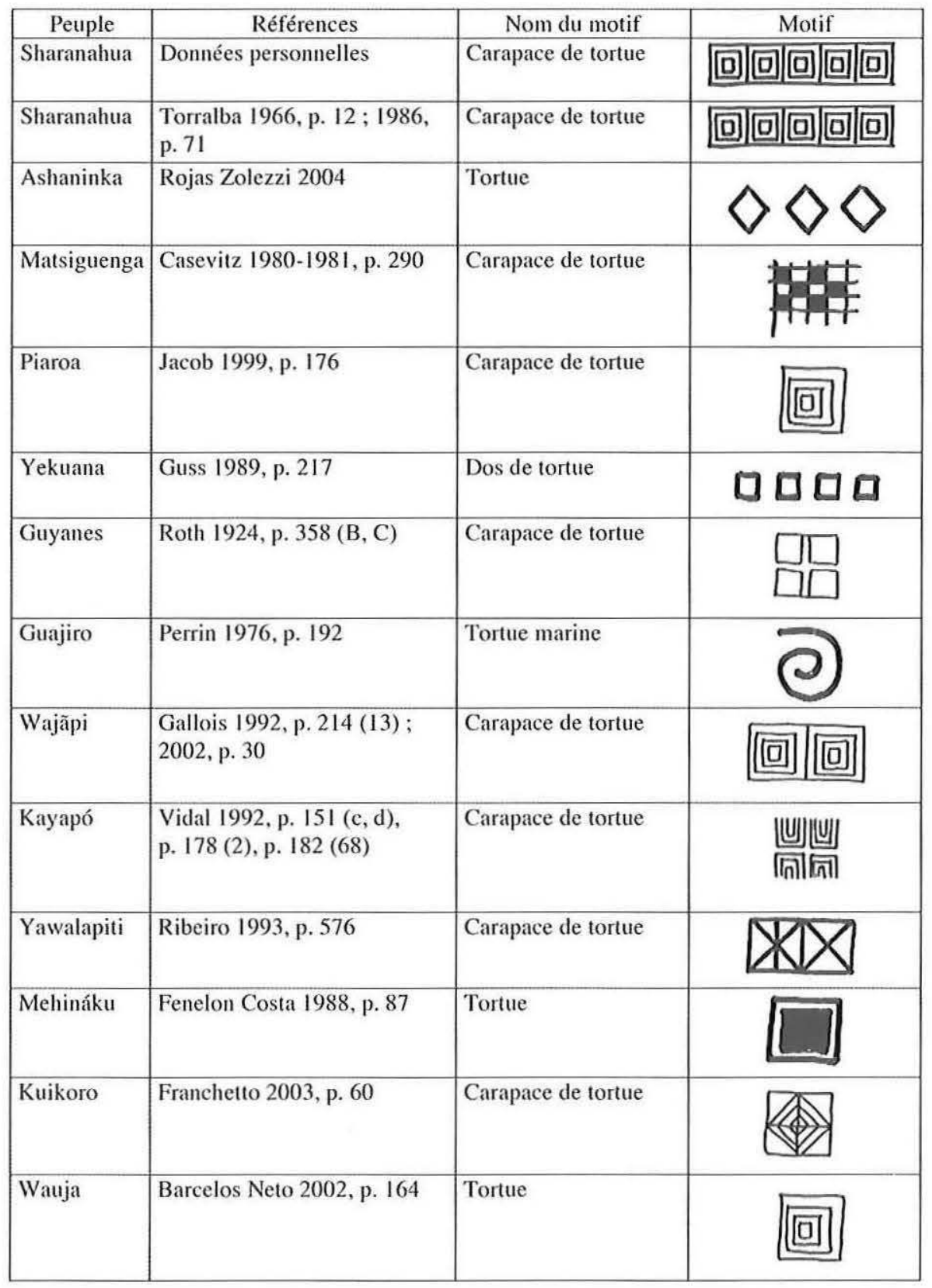

Fig. 8 - Le motif " tortue». 
treillis (Figure 9). Nous aurions pu également consacrer un tableau aux motifs " papillon » (séries de triangles dédoublés accolés à leurs sommets) et "dent » (séries de triangles), ainsi qu'à quelques autres ${ }^{11}$. On constate que les mêmes motifs correspondent à des noms semblables au sein de groupes culturellement et géographiquement très distants : chez les Pano d'Amazonie occidentale, chez les Arawak du piémont andin, chez les Jivaro, chez plusieurs groupes (en particulier Caribes) du nord de l'Amazonie, chez les Gê et les peuples du Xingu. Il faut préciser que cette recherche d'homologies est fortement limitée par des sources rares et incomplétes.

Que dire de cette surprenante homogénéité ? Écartons immédiatement l'hypothèse diffusionniste ; non qu'elle soit nécessairement fausse - elle apparaît, plus simplement, indémontrable. D'autant qu'elle esquive le problème de la stabilisation de telles associations entre motifs graphiques et noms spécifiques au cours de leur transmission.

Nous souhaiterions plutôt voir dans cette relative homogénéité panamazonienne un indice probant du caractère non figuratif des motifs graphiques. Nous avons déjà montré que les séries de motifs étaient susceptibles d'être construites à partir de lois de composition purement graphiques. Si l'on prend en compte à la fois cet engendrement autonome des séries de motifs et l'ubiquité amazonienne d'un bon nombre d'associations entre motif et nom, il devient possible d'imaginer que les noms ont été attribués aux motifs a posteriori. Le motif du «serpent " n'est pas réalisé pour figurer le serpent; il est nommé « serpent » dans la mesure où il fait penser au serpent. Chez les Jivaro, « le nom des motifs n'a qu'un rapport très indirect à leur référent ostensible ; et quand bien même on serait tenté de voir dans la combinaison de motifs composant une peinture une imitation stylisée d'un visage de félin ou d'anaconda, celle-ci, au dire même des Indiens, n'a rien d'iconique " (Taylor 2003, p. 239). Ces noms des motifs ne doivent donc pas être compris au sein d'une logique de la figuration. Ils ne résultent que d'une reconnaissance analogique, a posteriori, fondée sur une saillance visuelle commune ${ }^{12}$.

Dès lors, pourquoi les motifs sont-ils nommés ? Notre hypothèse est que si les motifs sont nommés, c'est afin de faciliter leur remémoration. En effet, les motifs n'ont de valeur, nous le montrerons bientôt, que dans la mesure où ils font partie d'un répertoire. Ils ne sont utiles qu'à partir du moment où ils se présentent comme une série de motifs discrets, comme un simple système de différences. La remémoration de tels stocks de motifs graphiques n'a rien d'évident ; toutefois, elle peut être nettement facilitée par une mise en correspondance systématique ou homologique de chacun des motifs avec un nom. On sait par ailleurs que ce genre de procédé est à la base de la plupart des mnémotechniques considérées comme efficaces : loin d'alourdir la mémoire, il structure le processus de la mémorisation ${ }^{13}$.

Si l'on prend au sérieux cet argument, on ne s'étonnera pas que les noms soient choisis de telle manière qu'ils permettent, par le biais d'une saillance 


\begin{tabular}{|c|c|c|c|}
\hline Peuple & Référence & Nom du motif & Motif \\
\hline Sharanahua & Données personnelles & $\begin{array}{l}\text { Arêtes du poisson } \\
\text { mojarra }\end{array}$ & $2 x \times x \times x \times x \times$ \\
\hline Sharanahua & Torralba 1966, p. 12 & $\begin{array}{l}\text { Écailles du poisson } \\
\text { boquichico }\end{array}$ & $2 x \times x \times x \times x$ \\
\hline Ashaninka & Rojas Zolezzi 2004 & $\begin{array}{l}\text { Écailles du poisson } \\
\text { kipaori }\end{array}$ & 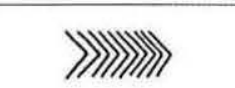 \\
\hline Matsiguenga & $\begin{array}{l}\text { Casevitz 1980-1981, p. } 282 \\
\text { (70) }\end{array}$ & Poisson koviri & \\
\hline Yanesha & $\begin{array}{l}\text { Duff-Tripp 1998, p. } 416 \\
\text { (achaque't) }\end{array}$ & Poisson carachama & \\
\hline Akawaio & Butt 1957, p. 50 (b) & Peau de poisson & \\
\hline Guyanes & Roth 1924, p. $358(\mathrm{~A})$ & Poisson & \\
\hline Wajãpi & $\begin{array}{l}\text { Gallois 1992, p. } 211(7) \text {; } \\
\text { 2002, pp. } 24-26\end{array}$ & Arêtes de poisson & $\mathrm{IIIII}$ \\
\hline Kayapó & Vidal 1992, p. 151 (i) & Arêtes de poisson & \\
\hline Bakairi & Von den Steinen 1940 & Poisson merexu & \\
\hline Wauja & $\begin{array}{l}\text { Coelho Pentado 1993, } \\
\text { pp. } 607,610\end{array}$ & Arêtes de poisson & \\
\hline Wauja & $\begin{array}{l}\text { Barcelos Neto 2002, p. } 164 \\
\text { (4) }\end{array}$ & Arêtes de poisson & \\
\hline Trumai & Monod Becquelin 1975 & Arêtes de poisson & \\
\hline Mehináku & Fenelon Costa 1988, p. 87 & Arêtes de poisson & \\
\hline Kuikoro & Franchetto 2003, pp. 54-56 & Arêtes de poisson & \\
\hline
\end{tabular}

FIG. 9 - Le motif « poisson ». 
visuelle commune, la remémoration d'un motif quelconque. C'est peut-être là l'unique fonction des noms des motifs : non pas l'indication d'une qualité figurative des dessins, mais une simple technique de remémoration d'une série d'éléments discrets. C'est ainsi que peut s'expliquer l'ubiquité de plusieurs associations entre motif et nom dans de nombreuses cultures amazoniennes très éloignées les unes des autres.

De ce point de vue, les répertoires graphiques amazoniens apparaissent comme des systèmes de différences relativement clos, dont l'aspect sémiotique n'a qu'une valeur mnémotechnique. Considérés comme tels, ils ne servent à rien. De ce fait, la notion de "répertoire graphique » n'a de valeur qu'analytique : elle n'intéresse pas leurs possesseurs qui se sont acharnés à le répéter aux nombreux ethnologues venus en quête de signification. C'est que les noms des motifs n'ont rien à voir avec leur utilisation - que celle-ci soit rituelle ou non. Lorsque les répertoires graphiques sont utilisés, ils prennent toute leur valeur aux yeux de leurs possesseurs ; c'est alors qu'il devient intéressant d'en parler. C'est ce que nous nous proposons de montrer ; toutefois, auparavant, nous reviendrons brièvement sur le champ sémantique des noms des motifs.

\section{LE CHAMP SÉMANTIQUE DES NOMS DES MOTIFS GRAPHIQUES}

Le lecteur aura remarqué que les noms de motifs listés dans les tableaux ne sont pas absolument identiques. Le motif " serpent ", par exemple, est dénommé de diverses manières : il peut s'agir de la queue de l'anaconda, d'une tête de serpent, des traces du serpent ou encore des dessins formés par les écailles du serpent. Pour rendre compte de ces variations, il faut revenir aux Sharanahua et observer de plus près le champ sémantique du terme cudu.

Comme dans bien d'autres langues d'Amazonie, ce terme ne se limite pas à une référence aux dessins corporels. Il permet également de dénoter toute inscription naturelle se signalant d'elle-même par son rythme régulier ou sa symétrie. Ainsi, les dessins sur la peau de l'anaconda ou sur la fourrure du jaguar seront nommés cudu ; même chose pour les traces régulières que laissent les petits oiseaux sur la plage. On pourrait même avancer qu'il existe quelque chose comme une catégorie implicite regroupant les animaux qui sont considérés cuduya, " couverts de dessins »; cette catégorie comprend le jaguar, certains serpents, plusieurs oiseaux et de nombreux poissons. On retrouve une telle polysémie au sein de la plupart des termes amazoniens dénotant les motifs graphiques ${ }^{14}$.

Dès lors, on peut décrire plus précisément la logique de dénomination qui est à l'œuvre dans les répertoires graphiques. Le point de départ consiste à repérer une saillance visuelle commune entre un motif graphique et un être quelconque. Il pourra donc s'agir de la forme globale d'un être, sa silhouette par exemple : le corps du serpent fera penser au zigzag. Mais il pourra s'agir aussi bien d'une 
partie corporelle d'un être : son coude, sa tête ou même ses os. Dans les deux cas, c'est le même procédé analogique qui est à l'œuvre. Deux autres cas exploitent, quant à eux, la polysémie du terme « dessin » : lorsque le nom du motif se réfère à la trace laissée par un être (ongle de tatou, trace du serpent) ou lorsqu'il dénote les dessins naturels dont s'ornent la peau ou la fourrure d'un être (dessin sur la peau du serpent, dessin sur la carapace de la tortue). On peut obtenir, à l'aide de ces quatre principes de dénomination, l'ensemble des noms utilisés par les répertoires graphiques amazoniens.

\section{LA PEINTURE FACIALE}

Lorsque les Sharanahua parlent de $c u d u$, ils ne se réfèrent pas seulement aux motifs que nous avons isolés. Ils désignent également les peintures achevées telles qu'on peut les observer sur les visages. Cette actualisation des motifs en peinture faciale doit être pensée en fonction de deux aspects : le premier est de l'ordre de la technique graphique, le second a trait à l'usage de ces dessins. Nous aborderons dès à présent le premier aspect.

Précisons tout d'abord que les cudu sharanahua n'ornent que les visages. Même si cela a pu être le cas dans le passé, les Sharanahua, à ce jour, ne décorent ni leur corps, ni leurs divers ustensiles de ces dessins. Leur actualisation doit donc tenir compte de leur unique support : le visage humain. L'observation de quelques peintures faciales fait apparaître clairement, d'une part, un axe de symétrie vertical, d'autre part, une ligne de séparation horizontale (Figure 10).

La présence de l'axe de symétrie vertical n'est pas très étonnante ; elle ne fait qu'exploiter la symétrie naturelle du visage. La ligne de séparation horizontale est plus singulière : elle permet de séparer des motifs de nature différente. Elle court des commissures de la bouche jusqu'aux oreilles, soulignant ainsi l'invisible mâchoire. C'est en général le premier trait que dessinent les peintres sharanahua. Toutefois, à Gasta Bala, deux femmes, d'un âge déjà avancé, n'avaient pas besoin de dessiner ce trait : il était déjà tatoué sur chacune de leurs joues. Ces tatouages, nommés tsitsu, forment comme les vestiges d'un passé où les femmes, au moment de leur puberté, étaient entraînées dans un rituel d'initiation dont une des phases consistait en l'imposition de ce tatouage. Le rituel a disparu ainsi que le sens de ces tatouages - si sens il fallait chercher. On remarquera simplement, après Philippe Erikson (1996, pp. 86, 318), la relative ubiquité de ce genre de tatouages chez les peuples pano, au début du siècle (Figure 11) ${ }^{15}$.

L'actualisation des motifs en dessins respecte donc, de manière conditionnelle, un axe de symétrie vertical et une ligne de séparation horizontale. Puis, chaque motif est élaboré en suivant les lois de la composition que nous avons signalées : symétries, translation et rotation. Le principe étant de remplir la partie inférieure du visage et de faire se répondre, par une symétrie en miroir, deux petits 


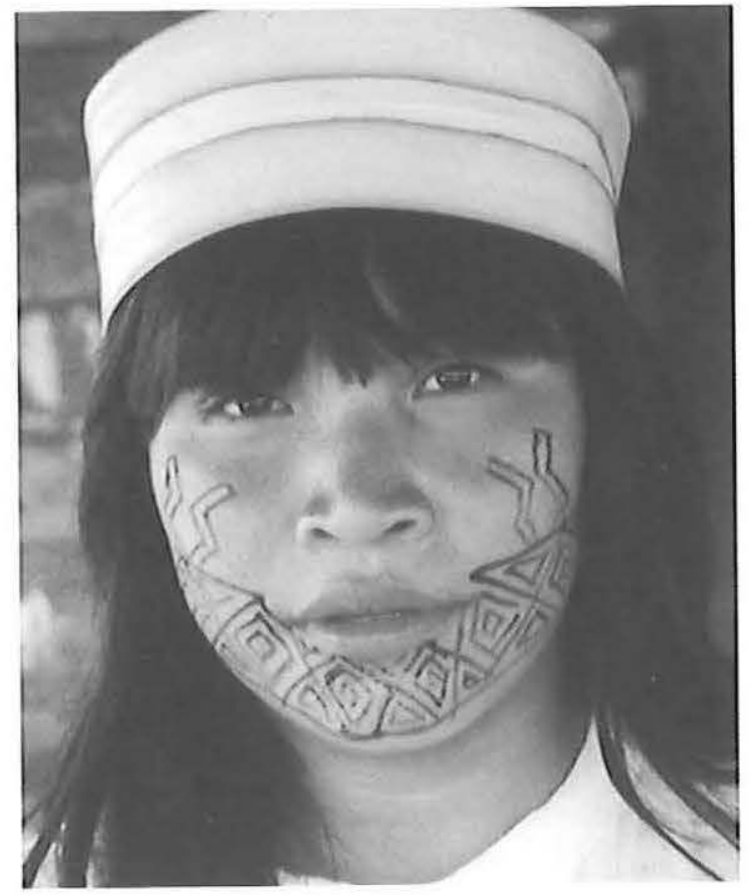

(a)

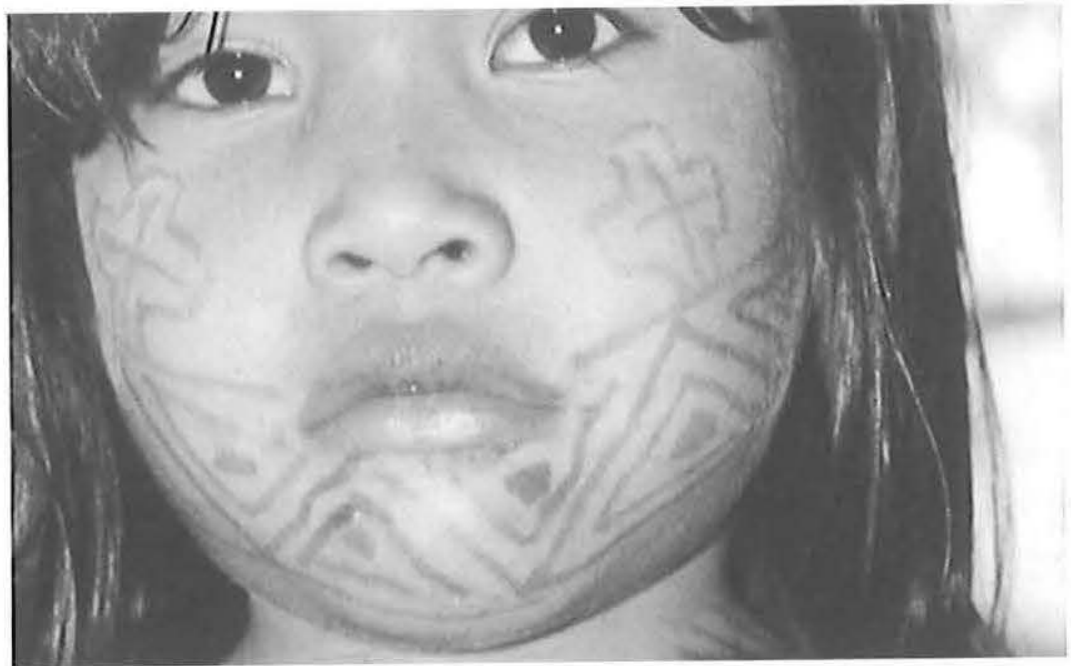

(b)

Fig. 10 -Exemples de peintures faciales sharanahua : (a) Ahuafuda (papillon) avec Ronohua cudu (anaconda), et Yora shao (squelette); (b) Iso Puti (coude de singe-araignée) et Fishi (étoile). 

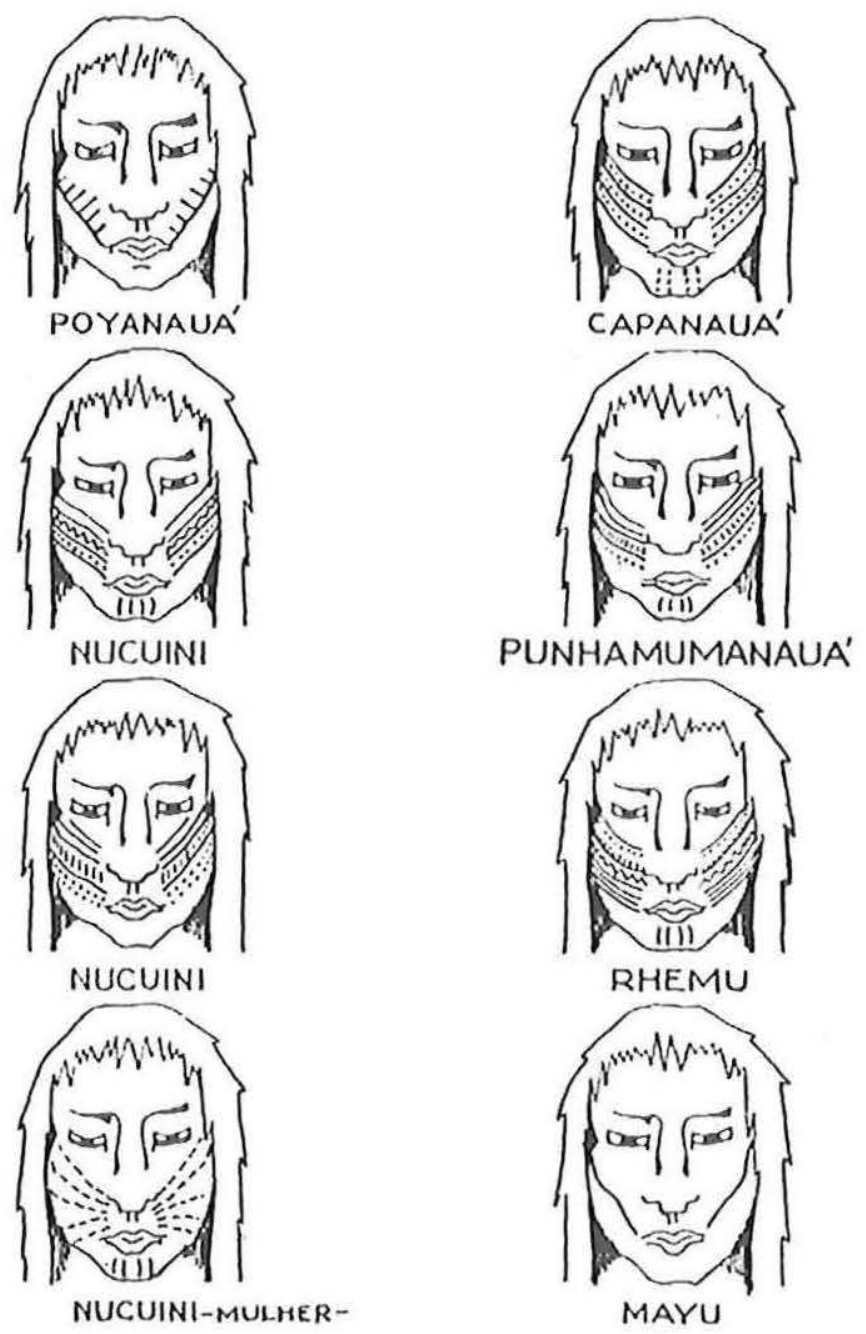

FIG. 11 - Tatouages pano (Oppenheim 1936, p. 144). 
motifs individualisés autour de l'axe du nez. Le peintre peut alors, à sa guise, ajouter quelques éléments : une ligne sur le front, des points autour des reliefs du visage, une élaboration de la ligne de séparation horizontale, etc.

\section{À QUOI SERVENT LES RÉPERTOIRES GRAPHIQUES ?}

Chez les Sharanahua, ce sont surtout les femmes qui se peignent le visage. À l'occasion, un homme pourra certes le faire, mais ce sera souvent sans y prendre soin et, de préférence, en se recouvrant la partie inférieure du visage d'un aplat de génipa (le verbe exprimant une telle action n'est alors plus /cudu-/, « dessiner des motifs ", mais /raish-/, " remplir une surface »). Les femmes, quant à elles, se peignent assez souvent, sans occasion particulière, lorsqu'il y a du génipa, du roucou ou du maquillage.

Nous avons montré que les noms des motifs graphiques des Sharanahua n'avaient d'autre fonction que mnémotechnique : ils servent à faciliter la mémorisation d'une série d'éléments discrets. Dès lors se pose une question : pourquoi a-t-on besoin de mémoriser une série de motifs graphiques? À quoi cela sert-il ? Pourquoi réaliser de telles peintures faciales? Cette question obsède souvent l'ethnologue qui la pose et la repose, en en variant sans cesse la forme, jusqu'à l'épuisement ou l'exaspération de ses interlocuteurs. En ce qui concerne les Sharanahua, nous avons obtenu pour l'essentiel ce genre de réponses : «On se peint parce que l'on vient de trouver du génipa dans la forêt » ou « On se peint pour être belles ». Il semble donc, à première vue, que les Sharanahua n'aient pas grand-chose à nous dire sur l'utilisation de leur répertoire graphique. En est-il de même ailleurs?

Réfléchissons déjà sur ce à quoi pourraient servir de tels répertoires. Chaque nom de motif pourrait, par exemple, être motivé et faire référence à un nom clanique. Le motif " tortue » serait alors porté par les membres du clan de la tortue. Une telle logique sémiotique est présente chez les Warlpiri d'Australie, par exemple, où « chaque clan possède une mosaïque de losanges unique, des petits aux plus allongés, aux origines mythiques diverses. Ces polygones représentent par exemple les cellules de la ruche d'abeille pour les Gupapuyngu et les écailles du crocodile brûlé par le feu pour les Gumatj. [...] Chaque tracé est associé à un lieu et à la signature d'un clan » (Glowczewski et al. 2005, pp. 23-24). Mais si, en Australie, un motif discret est nécessairement associé, en vertu de son nom et en contexte rituel, à un clan, un ancêtre, un paysage, un chant, une danse et certains objets (Morphy 2005) ${ }^{16}$, nous n'avons toujours pas trouvé, en Amazonie, de cas similaires. De toute évidence, il faut voir dans ce fait un nouvel argument tendant à montrer que, relativement à leur usage, les associations amazoniennes entre motifs et noms sont arbitraires.

Cependant, l'ethnographie amazonienne, si on l'interroge de ce point de vue, fournit une donnée pertinente : les répertoires graphiques servent, dans certains 
contextes (éventuellement rituels), à rendre visible une relation sociale au détriment d'autres types de relation. Selon la formule synthétique d'Anne Christine Taylor (2003, p. 245), à propos des Jivaro, « les peintures sont la trace visuelle d'un mode de relation à autrui ». Cela signifie qu'un motif, une fois peint sur un corps, permet, d'une part, d'identifier ce corps aux autres corps ornés du même motif et, d'autre part, de différencier ce corps de ceux qui ne sont pas ornés du même motif. C'est là une fonction très simple qui ne repose pas sur le sémantisme des motifs, mais qui nécessite, dans une certaine mesure, la mémorisation d'une pluralité de motifs. Cette exhibition matérielle des relations à autrui repose donc sur le contraste, purement graphique, entre chacun des motifs, et non sur la signification de leurs noms.

Ces relations sélectionnées d'identité et de différence peuvent concerner des groupes d'amplitude extrêmement variable. Le cas le plus complexe, et peut-être le mieux documenté, est celui des Kayapó-Xikrin où ces relations passent entre une multitude de sous-groupes définis par le sexe, la classe d'âge, la position rituelle et quelques autres éléments distinctifs (Vidal 1992). Les différences entre les motifs graphiques permettront de rendre visibles les différences entre ces sous-groupes. Remarquons que, de ce point de vue, et cela reste vrai de tous les usages rituels des dessins, le répertoire graphique possède la même fonction que les parures corporelles (plumes, colliers, ceintures, etc.) (Verswijver 1982-1983).

Il existe toutefois de nombreuses variations sur ce même principe. Pour rester chez les Gê, les Xerente utilisent leurs motifs pour rendre visible une différence entre moitiés exogamiques (Lopes da Silva et Farias 1992) ${ }^{17}$. Les Guajiro, quant à eux, expriment ainsi une simple différence de genre (Perrin 1976, p. 212). La mythologie waiwai met en scène une série de clans qui se différencient les uns des autres à l'aide de dessins (Fock 1963, p. 56) - comme la mythologie sharanahua raconte l'origine des divers "clans » en leur attribuant à chacun une parure corporelle distincte, mais non sémantiquement motivée.

À l'extrémité de ce spectre, on trouve plusieurs groupes amazoniens où la seule différence qui vaille la peine d'être exprimée est celle qui passe entre les individus. Dans ce dernier cas, chaque membre du groupe tiendra à ce que sa propre peinture faciale soit unique, différente de toutes les autres. C'était là une exigence à laquelle tenaient fortement toutes les femmes sharanahua, mais c'est une situation que l'on retrouve, semble-t-il, dans de nombreux groupes amazoniens - par exemple, chez les Jivaro (Taylor 2003) ${ }^{18}$.

Finalement, les Sharanahua insistaient sur un autre aspect de leurs peintures faciales : elles étaient les leurs, elles appartenaient aux odi coin, « nous, par opposition aux autres ». C'est ainsi qu'ils affirmaient que leurs motifs différaient fondamentalement de ceux de leurs voisins Cashinahua : ces derniers, selon les Sharanahua, n'utilisaient qu'une grecque, véritable motif originel répété à l'envi ${ }^{19}$. En aucun cas, les Sharanahua n'utiliseraient ce motif qu'ils considèrent comme un motif " carapace de tortue » manqué. Il s'agissait là d'une ultime 
logique de différenciation rendue visible, séparant un groupe d'un autre, comme le faisaient peut-être, fut un temps, les tatouages.

Dans tous les cas, le répertoire sert à rendre visibles des différences sociales à l'aide de son propre système de variations graphiques - différences certes extrêmement hétérogènes qui peuvent osciller entre une simple distinction superficielle et un mode d'individuation fort pensé comme une identification. Les divergences entre les motifs, et non le sémantisme de leur nom, sont alors analogues à celles entre groupes ou individus, dans un contexte plus ou moins ritualisé. Le répertoire est donc un outil dont l'utilisation, d'une grande souplesse, est à géométrie variable, s'adaptant à une amplitude qui va du groupe en son entier à l'individu. L'aspect cognitif de ce répertoire, l'association terme à terme d'un motif graphique à un nom, n'a de valeur que mnémotechnique : il permet de conserver une série de différences graphiques ${ }^{20}$.

\section{Catégories et innovations}

En partant d'une description des peintures faciales sharanahua, nous avons essayé de démontrer l'existence d'une catégorie pan-amazonienne : le répertoire graphique. Ces répertoires sont des listes de motifs graphiques discrets associés terme à terme à des noms spécifiques. Ces motifs ne sont en aucun cas figuratifs, la valeur de leur nom est analogique et mnémotechnique. Les répertoires sont donc de purs systèmes de différences. Ils peuvent être utilisés de manières variées afin d'exprimer d'autres différences, appartenant à une autre logique relationnelle, qui leur préexiste. Nous disposons maintenant d'une définition solide de la catégorie amazonienne se rapportant à ces artefacts que sont les répertoires graphiques. Il sera possible dorénavant de reprendre à nouveau l'étude des figurations amazoniennes qui forment sans doute une autre catégorie, puis celle de ces étranges et mal nommés « dessins spontanés » dont regorgent les archives de nombreux ethnologues et qui constituent probablement encore une nouvelle catégorie iconographique, mixte celle-ci.

Si le champ de recherche s'ouvre ainsi vers une comparaison entre les diverses catégories d'artefacts hétérogènes, graphiques ou iconographiques, d'une même culture, il peut également être développé en étudiant les multiples formes que revêt une même catégorie dans plusieurs contextes culturels. Une première approche de cette problématique consisterait alors à se pencher sur la manière dont les peuples disposant de répertoires graphiques ont appréhendé l'écriture, cet autre ensemble de signes graphiques non figuratifs fonctionnant, entre autres, comme une mémoire externe. De ce point de vue, on peut observer que les populations amazoniennes ont développé deux interprétations récurrentes de l'écriture importée par les étrangers ${ }^{21}$. La première est, si l'on veut, une catégorisation par défaut : un grand nombre de peuples amazoniens ont choisi de désigner l'écriture 


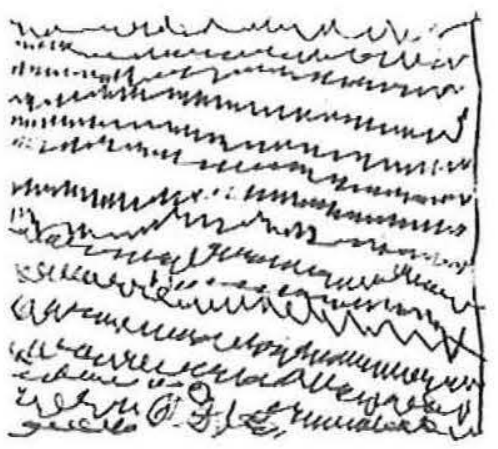

(a)
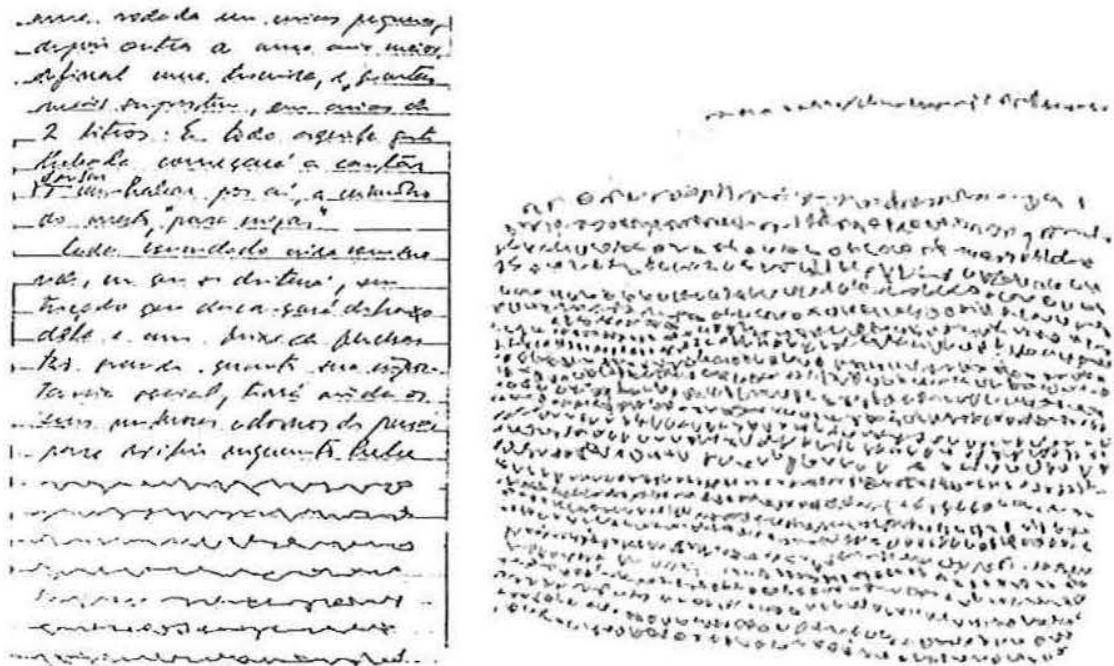

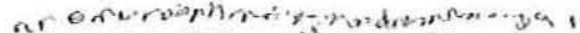

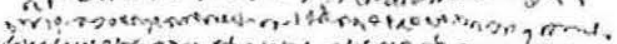

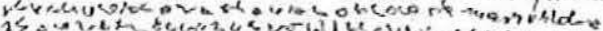

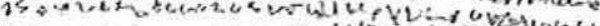

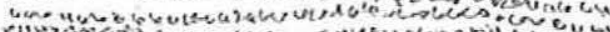

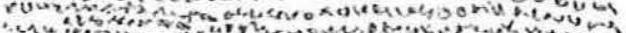

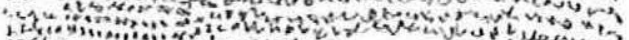
t.

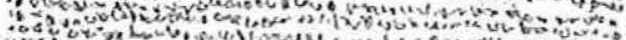

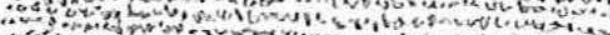

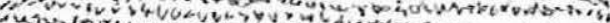

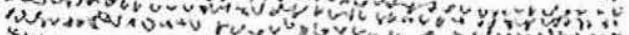
sust

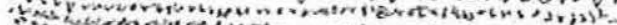

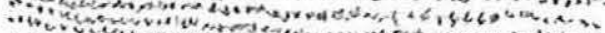

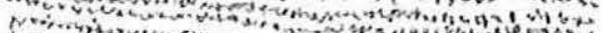

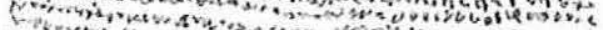

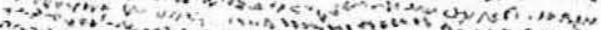

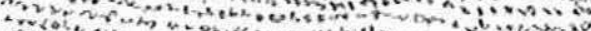

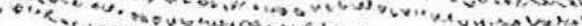
is

(b)

FIG. 12 - Lignes d'écriture amazoniennes : nambikawara (a), urubus-kaapor (b), matis (c) (d'après Lévi-Strauss 1964, p. 331 ; Ribeiro 2002, p. 172 ; Erikson 1996, p. 309).

à l'aide du terme même qui leur permettait de dénoter leurs répertoires graphiques. Ainsi, chez les Sharanahua, cudu signifie aussi bien " peinture corporelle » qu'« écriture » ${ }^{22}$. La seconde interprétation est proprement graphique : ce sont ces « lignes d'écriture » que plusieurs ethnologues ont recueillies (Figure 12). Ces lignes, successions de zigzags rythmées, étaient le résultat que pouvaient obtenir certains peuples amazoniens lorsqu'ils décidaient d'imiter l'écriture des étrangers. Elles représentaient, peut-être, à la fois une manière de reconnaître une certaine affinité, phénoménologique, entre répertoire graphique et écriture, et une 
façon de les opposer tous deux à l'ensemble distinct des figurations. Les peuples amazoniens disposaient ainsi de catégories déjà constituées qui leur permirent d'appréhender l'écriture venue des étrangers. *

* Manuscrit reçu en juin 2006, accepté pour publication en décembre 2006.

\section{Notes}

1. On trouvera une telle analyse dans Déléage (2005, pp. 39-98).

2. Les anthropologues étudiant l'art des Aborigènes d'Australie utilisent une dichotomie semblable à celle des Sharanahua : ils distinguent nettement, d'une part, les « figurations » et, d'autre part, les images « non iconiques » ou « géométriques » (Morphy 1989; Taylor 1989).

3. On trouve une manière semblable d'envisager le motif de la "croix » chez les Yekuana (Guss 1989 : le motif Wanadi Motai est composé de deux chevrons accolés) et, peut-être, chez les Wauja (Coelho Pentado 1993, p. 609, motif 3C, et p. 611, motif 4B; Barcelos Neto 2002, p. 164, motif 9).

4. Voir néanmoins Vidal et Müller (1987).

5. Plusieurs auteurs (Roe 1989 ; Myers 2002) s'accordent pour voir dans les dessins curvilignes des Shipibo (maya quënëa) une invention relativement récente. Voir aussi, pour les Yine, Gow (1999, p. 233).

6. Voir aussi, pour les Wauja, Barcelos Neto (2001, p. 197).

7. Remarquons que le sixième motif nommé des Wajàpi, "crapaud ", est présent, avec le même nom, dans le répertoire graphique cashinahua (Lagrou 1998), voisins des Sharanahua.

8. Ces motifs ont diverses modalités d'exécution : dessins corporels, tatouages, vannerie, tissage, etc. C'est en partie pour cette raison que le terme " répertoire graphique ", qui introduit un certain degré d'abstraction en ne prenant pas en compte cette diversité d'exécution, nous semble pertinent.

9. Voir aussi pour les Shipibo, Ríos Cairuna (2003) : l'identification 19, une série de losanges nommée " dessin du boa " et l'identification 40, une ligne en zigzag nommée " silhouette de boa ».

10. Voir aussi, pour les Shipibo, Soria Casaverde (2004, p. 27 figure 1) et, pour les Panare, Henley et Mattéi-Müller (1978, pp. 92-94), dont l'analyse, découverte tardivement, concorde parfaitement avec notre hypothèse.

11. De nombreuses sources présentent soit des motifs similaires sans leurs noms (Schultz 1963 ; Kensinger 1975 ; Baer 1994 ; Bianchi 1982 ; Verswijver 1992 ; Roe 1989), soit des noms similaires sans les motifs correspondants (Abreu 1941 ; Carid Naveira 1999; Ahlbrinck 1956; Gregor 1977 ; Baldus 1970 ; Perrin 1979; Valenzuela et Valera Rojas 2005). Les Asurini, quant à eux, construisent leurs motifs à partir d'une logique de décomposition plutôt que de composition; la plupart des noms de leurs motifs se rapportent néanmoins à la tortue (Vidal et Müller 1987; Müller 1990, 1992). On retrouve le motif " papillon " chez les Sharanahua, les Cashinahua, les Ashaninka, les Matsiguenga, les Wajàpi, les Wauja, les Trumai et les Kayapo. Le motif " dent » est attesté chez les Sharanahua, les Wauja, les Mehinaku et les Trumai. Il faut remarquer qu'il existe au nord de l'Amazonie un vaste ensemble homogène où les motifs sont d'une nature beaucoup plus figurative ; il s'agit soit de motifs de vannerie, soit de pcintures corporelles (Ribeiro 1987; Hurault 1968; Van Velthem 1992; KochGrunberg 1982; Fock 1963 ; Yde 1965 ; Guss 1989 ; Roth 1924 ; Reichel-Dolmatoff 1985, 1987). Même chose chez les Miraña (Karadimas 2000-2001). Ces ensembles ne rentrent pas dans le cadre de notre analyse.

12. En insistant ainsi sur la dénomination a posteriori des motifs, nous ne formulons ni une hypothèse phylogénétique, ni une hypothèse ontogénétique ; nous ne souhaitons que souligner l'autonomie graphique du répertoire vis-à-vis de la dénomination de chacun de ses motifs.

13. Nous renvoyons, d'une part, aux nombreux travaux sur l'art de la mémoire (Yates 1975; Bolzoni 2005) et, d'autre part, à sa « redécouverte » aussi bien par un individu isolé doté d'une capacité 
mnémonique surprenante (Luria 1987) que par de nombreuses écoles d'interprétariat contemporaines. Remarquons toutefois que, ce qu'il s'agit de mémoriser dans le cas qui nous occupe, ce sont des dessins et non des discours.

14. De ce point de vue, on pourra consulter, par exemple, Gow (1999).

15. Voir également Oppenheim (1936, p. 144) et Tessmann (1999, planche 64).

16. Voir aussi, par exemple, Poirier (1992) et Dussart (1997).

17. Les Canela, quant à eux, auraient utilisé, au cours des rituels de puberté, six emblèmes peints au génipa exprimant chacun l'appartenance à un clan (Crocker 1986, p. 26).

18. Cette différence individuelle exprime aussi, dans le cas jivaro, une identité surnaturelle unissant un individu à son arutam. Ce genre d'identification, présent au sein de rituels de type chamanique ou de mythes, est lié aux dessins en général, et non aux noms de chacun des motifs du répertoire. Au-delà de l'exemple jivaro, le lecteur américaniste sera sans doute étonné de notre silence sur l'idée selon laquelle les dessins participent à la fabrication du corps de celui qui les porte et donc à un processus de transformation ontologique ou de constitution d'une identité. C'est que, d'une part, il est difficile de généraliser cette logique dans la mesure où l'usage des dessins s'eflectue dans le cadre d'un continuum qui va du contexte le plus quotidien au dispositif le plus précisément ritualisé, et que, d'autre part, il nous est apparu que le sémantisme des motifs n'entretenait pas de rapport avec cette logique essentiellement rituelle (le motif " tortue » ne transforme pas son porteur en tortue).

19. Il est vrai que ce motif semble très important pour les Cashinahua (Schultz 1963 ; Kensinger 1975). On remarquera d'ailleurs que les Cashinahua représentent le yaminahua kene, c'est-à-dire le " motif des Sharanahua ", sous la forme d'une variation sur le thème du chevron (Lagrou 1998) - ce qui pourrait laisser penser que la grecque est aux Cashinahua ce que le chevron est aux Sharanahua.

20. Notre analyse est, de ce point de vue, tout à fait comparable à cette remarque d'Andrew et de Marilyn Strathern sur les noms des motifs des peintures faciales du Mount Hagen : " The names are mnemonics, which label the patterns used and enable them to be listed and stored in people's minds” (Strathern et Strathern 1971, p. 108).

21. Nous ne prenons pas ici en compte les interprétations mythiques et chamaniques de l'écriture qui feront l'objet d'un autre travail.

22. Le même terme dénote les motifs graphiques et l'écriture chez les Pano, les Yine, les Arawak sub-andins, les Jivaro, les Siona, les Ticuna, les Guajiro, les Wayana, les Wajàpi, les Trumai, les Asurini, les Kayabi, les Kamayura, les Tapirapé, etc.

\section{RÉFÉRENCES BIBLIOGRAPHIQUES}

\section{Abreu João Capistrano de}

1941 Rà-txa Hu-ni-ku-i. Grammatica, textos e vocabulario caxinauas, Sociedade Capistrano de Abreu/Livraria Briguiet, Rio de Janeiro [1914].

AhLBRINCK Willem

1956 Encyclopédie des Caraibes, s. n., Paris [1931].

BAER Gerhard

1994 Cosmologia y shamanismo de los Matsiguenga (Perú Oriental), Abya-Yala, Quito.

\section{BaLdus Herbert}

1970 Tapirape, tribo tupi no Brasil central, Companhia Editora Nacional, Sao Paulo. 
Barcelos Neto Aristoteles

2001 "Apontamentos para uma iconografia historica Xinguana », in Bruna Franchetto et Michael Heckenberger (éds), Os povos do Alto Xingu. Historia e cultura, UFRJ, Rio de Janeiro, pp. 193-218.

2002 A arte dos sonhos. Uma iconografia amerindia, Assirio \& Alvim, Lisboa.

\section{BIANCHI César}

1982 Artesanias y técnicas shuar, Ediciones Mundo Shuar, Quito.

BoAs Franz

2003 L'Art primitif, A. Biro, Paris [1927].

Bolzoni Lina

2005 La Chambre de la mémoire. Modèles littéraires et iconographiques à l'âge de l'imprimerie, Droz, Genève.

Butr Audrey

1957 "The mazaruni scorpion. A study of the symbolic signifiance of tatoo patterns among the Akawaio ", Timehri, 36, pp. 40-55.

Carid Naveira Miguel

1999 Yawanawa : da guerra à festa, dissertação de mestrado, Universidade Federal de Santa Catarina, Florianópolis.

CASEvitz France-Marie

1980-1981 "Inscriptions, un aspect du symbolisme matsiguenga », Journal de la Société des Américanistes, 67, pp. 261-295.

Coelho Pentado Vera

1993 "Motivos geométricos na arte Uaura », in Vera Penteado Coelho (éd.), Karl von den Steinen : um século de antropologia no Xingu, EDUSP/FADESP, Sao Paulo, pp. 591-630.

Crocker William H.

1986 "Canela body painting », Latin American Literature and Arts, 36, pp. 24-26.

DéléAge Pierre

2005 Le Chamanisme sharanahua. Enquête sur l'apprentissage et l'épistémologie d'un rituel, thèse de doctorat, École des hautes études en sciences sociales, Paris.

\section{Dussart Françoise}

1997 "A body painting in translation ", in Marcus Banks et Howard Morphy (éds), Rethinking Visual Anthropology, Yale University Press, New Haven/Londres, pp. 186-202.

DuFF-Tripp Martha

1998 Diccionario yamesha' (amuesha) - castellano, ILV, Lima.

ERIKsON Philippe

1996 La griffe des aïeux. Marquage du corps et démarquages ethniques chez les Matis d'Amazonie, Peeters/SELAF, Paris. 


\section{Fenelon Costa Maria Heloisa}

1988 O mundo dos Mehináku e suas representações visuais, Editora Universidade de Brasília, Brasilia.

Fock Niels

1963 Waiwai, religion and society of an Amazonian tribe, National Museum, Copenhague.

Franchetto Bruna et Mestres Karib do Alto Xingu

2003 Ikú ügühütu higei. Arte gráfica dos povos Karib do Alto Xingu, Museu do Indio/FUNAI, Rio de Janeiro.

Gallors Dominique Tilkin

1992 "Arte iconográfica Waiãpi », in Lux Vidal (éd.), Grafismo indigena : estudos de antropologia estética, Studio Nobel/FAPESP/EDUSP, Sao Paulo, pp. 209-230.

2002 Kusiwa. Pintura corporal e arte gráfica wayãpi, Museu do IndioFUNAI/APINA/CTI/NHII-USP, Rio de Janeiro.

Glassner Jean-Jacques

s. d. $\quad$ "Le corps écrit, la victime dans le sacrifice divinatoire en Mésopotamie » [à paraître].

Glowczewski Barbara, Jessica De Largy Healy et les artistes de Lajamanu et Galiwin'ku

2005 Pistes de rêves. Voyage en terres aborigènes, Éditions du Chêne, Paris.

Gow Peter

1999 «Piro designs : painting as meaningful action in an Amazonian lived world ", Journal of the Royal Anthropological Institute, 5 (2), pp. 229-246.

\section{Gregor Thomas}

1977 Mehinaku. The drama of daily life in a Brazilian village, The University of Chicago Press, Chicago/Londres.

Guss David

1989 To weave and sing. Art, symbol, and narrative in the South American rain forest, University of California Press, Berkeley.

Henley Paul et Marie-Claude Mattéi-Muller

1978 "Panare basketry : means of commercial exhange and artistic expression ", Antropológica, 49, pp. 29-130.

HolbraAd Martin

2003 «Estimando a necessidade : os oráculos de ifá e a verdade em Havana », Mana, 9 (2), pp. 39-77.

Hurault Jean

1968 Les Indiens Wayana de la Guyane française. Structure sociale et coutume familiale, ORSTOM, Paris. 
JACOB Wenzel (éd.)

1999 Orinoco-Parima. Indian societies in Venezuela. The Cisneros Collection, Hatje Cantz Publishers, Ostfildern-Ruit.

KaRADIMAS Dimitri

2000-2001 «Singes, raies et étoiles entre les Andes et l'Amazonie : perspectives comparatives dans l'iconographie amérindienne équatoriale ", Bulletin de la Société Suisse des Américanistes, 64-65, pp. 83-96.

KARSTEN Rafael

1935 The head-hunters of Western Amazonas: the life and culture of the Jibaro indians of Eastern Ecuador and Peru, Finska vetenskaps-societeten, Helsingfors.

Kensinger Kenneth (éd.)

1975 The Cashinahua of Eastern Peru, Haffenreffer Museum of Anthropology, Philadelphia.

KOCH-GRUNBERg Theodor

1982 Del Roraima al Orinoco, Tomo III, Ediciones del Banco central de Venezuela, Caracas [1924].

LAGRou Elsje

1998 "Visions that the plants gave us. Cashinahua drawings and their role in the vision quest » [http://web.stlawu.edu/gallery/elagrou.htm].

Lévi-Strauss Claude

1964 Le Cruet le cuit, Plon, Paris.

LOPES Da Silva Aracy et Agenor T. P. Farias

1992 "Pintura corporal e sociedade : os “partidos" Xerente », in Lux Vidal (éd.), Grafismo indigena: estudos de antropologia estética, Studio Nobel/ FAPESP/EDUSP, Sao Paulo, pp. 89-116.

LURIA Aleksandr Romanovicz

1987 The Mind of a Mnemonist, Harvard University Press, Cambridge [1968].

Monod BeCQuel.in Aurore

1975 La Pratique linguistique des indiens Trumai, SELAF, Paris.

1993 "O homem apresentado ou as pinturas corporais dos indios trumais », in Vera Penteado Coelho (éd.), Karl von den Steinen : um século de antropologia no Xingu, EDUSP/FADESP, Sao Paulo, pp. 511-562.

MORPHY Howard

1989 "On representing ancestral beings », in Howard Morphy (éd.), Animals into art, Unwin Hyman, Londres, pp. 144-160.

2005 "Style and meaning : Abelam art throught Yolngu eyes », Res, 47, pp. 209230.

Muller Regina

1990 Asurini do Xingu. Historia e arte, Unicamp, Sao Paulo.

1992 " Tayngava, a noção de representação na arte gráfica Asurini do Xingu », in Lux Vidal (éd.), Grafismo indigena : estudos de antropologia estética, Studio Nobel/FAPESP/EDUSP, Sao Paulo, pp. 231-248. 


\section{MYers Thomas}

2002 «Looking inward : the florescence of Conibo/Shipibo art during the rubber boom ", Bonner Amerikanistische Studien, 36, pp. 127-140

\section{OPPENHEIM Victor}

1936 «Notas etnograficas sobre os indigenas do Alto Jurua (Acre) e valle do Ucayali (Peru) ", Anais da Academia Brasileira de Sciencias, VIII, pp. 145155.

\section{Pellizzaro Siro}

1993 "Técnicas y estructuras familiares de los Shuar », in Juan Bottasso (éd.), Los Salesianos y la Amazonia. Tomo II. Relaciones etnográficas y geográficas, Abya-Yala, Quito, pp. 245-324

PERrin Michel

1976 Le Chemin des indiens morts, Payot, Paris.

1979 Sükuaitpa Wayuu. Los guajiros: la palabra y el vivir, Fundación La Salle, Caracas.

\section{PoIrier Sylvie}

1992 «Cosmologie, personne et expression artistique dans le désert occidental australien », Anthropologie et sociétés, 16 (1), pp. 41-58.

\section{Reichel-DolmatoFf Gerardo}

1985 Basketry as metaphor: Arts and crafts of the Desana indians of the Northwest Amazon, Occasional Papers of the Museum of Cultural History $\mathrm{n}^{\circ} 5$, University of California, Los Angeles.

1987 Shamanism and art of the Eastern Tukanoan Indians, E. J. Brill, Leiden.

Ribeiro Berta G.

1987 "Desenhos semânticos e identitade étnica : o caso Kayabí », in Berta G. Ribeiro (éd.), Suma etnológica brasileira 3, Arte india, VOZES/FINEP, Petrópolis, pp. 265-286.

1993 «Os padrões ornamentais do trançado e a arte decorativa dos indios do Alto Xingu ", in Vera Penteado Coelho (éd.), Karl von den Steinen : um século de antropologia no Xingu, EDUSP/FADESP, Sao Paulo, pp. 563-590.

RiBEIRo Darcy

2002 Carnets indiens. Avec les Indiens Urubus-Kaapor, Brésil, Plon, Paris.

Ríos CaIruna Laureano

2003 Diseños Shipibo-Conibo, Seminario de Historia Rural Andina, UNMSM, Lima.

Ros Peter

1980 «Art and residence among the Shipibo Indians of Peru : a study in microacculturation ", American Anthropologist, 82 (1), pp. 42-71.

1989 "Of rainbow dragons and the origins of designs : the Waiwai and the Shipibo Ronin Ehua ", Latin American Indian Literatures Journal, V (1), pp. 1-67. 


\section{Rojas Zolezzi Enrique Carlos}

$2004 \quad$ Mythes de la création du monde, représentations du gibier et des plantes cultivées et définition de l'ordre social traditionnel chez les Campa Ashaninka de l'Orient péruvien, thèse de doctorat, École des hautes études en sciences sociales, Paris.

\section{Rотн Walter Edmund}

1924 "Introductory study of arts, crafts and customs of the Guiana Indians ", in Thirty-Eight annual report of the Bureau of American Ethnology of the Smithsonian Institution 1916-1917, Government Printing Office, Washington, pp. 25-745.

\section{SCHмIDT Max}

1942 Estudos de etnologia brasileira. Peripécias de uma viagem entre 1900-1901. Seus resultados etnológicos, Companhia Editora Nacional, Sao Paulo.

Schultz Harald

$1963 H o m b u$, Éditions Vilo, Paris.

Soria Casaverde María Belén

2004 Introducción al mundo semiótico de los diseños shipibo conibo, Seminario de Historia Rural Andina, UNMSM, Lima.

STEINEn Karl von den

1940 Entre os aborigenes do Brasil central, Departamento de cultura, São Paulo [1893].

Strathern Andrew et Marilyn Strathern

1971 Self-Decoration in Mount Hagen, Gerald Duckworth \& Co, Londres.

TAYLor Anne-Christine

2003 «Les masques de la mémoire. Essai sur la fonction des peintures corporelles jivaro ", L'Homme, 165, pp. 223-248.

TAYLOR Luke

1989 "Seeing the "inside" : Kunwinjku paintings and the symbol of the divided body ", in Howard Morphy (éd.), Animals into art, Unwin Hyman, Londres, pp. 371-389.

\section{Tessmann Günter}

1999 Los indigenas del Perú Nororiental, Abya Yala/Internaciones, Quito [1930].

TORralba Adolfo

1966 "Pintura sharanahua », Misiones Dominicanas del Perú, XLVII (270), pp. 10-13.

1986 Sharanahua, Secretariado de Misiones Dominicanas, Madrid/Lima.

Valenzuela Pilar et Augustina Valera Rojas

2005 Koshi shinanya ainbo. El testimonio de una mujer shipiba, Universidad Nacional Mayor de San Marcos, Lima. 
VAN Velthem Lucia Hussak

1992 "Das cobras e lagartas: a iconografia Wayana », in Lux Vidal (éd.), Grafismo indigena: estudos de antropologia estética, Studio Nobel/ FAPESP/EDUSP, Sao Paulo, pp. 53-66.

VersivijVer Gustaaf

1982-1983 "Essai sur l'usage de la parure chez les indiens Kaiapo du Brésil central », Bulletin du Musée d'ethnographie de Genève, 25-26, pp. 23-44.

1992 The Club-Fighters of the Amazon. Warfare among the Kaiapo Indians of Central Brazil, Rijksuniversiteit, Gent.

ViDAL LuX

1992 "A pintura corporal e a arte gráfica entre os Kayapó-Xikrin do Cateté », in Lux Vidal (éd.), Grafismo indigena : estudos de antropologia estética, Studio Nobel/FAPESP/EDUSP, Sao Paulo, pp. 143-190.

VidAL Lux (éd.)

1992 Grafismo indigena: estudos de antropologia estética, Studio Nobel/FAPESP/EDUSP, Sao Paulo.

VIDAL Lux et Regina A. P. Muller

1987 "Pintura e adornos corporais », in Berta G. Ribeiro (éd.), Suma etnológica brasileira 3, Arte india, VOZES/FINEP, Petrópolis, pp. 119-148.

Washburn Dorothy K. et Donald W. Crowe

1988 Symmetries of culture. Theory and practice of plane pattern analysis, University of Washington Press, Seattle.

YATES Frances

1975 L'art de la mémoire, Gallimard, Paris [1966].

YDE Jens

1965 Material culture of the Waiwai, The National Museum of Copenhagen, Copenhague. 